\title{
Estimation of finite population mean by using minimum and maximum values in stratified random sampling
}

Umer Daraz

Quaid-i-Azam University, umerdaraz09@yahoo.com

Javid Shabbir

Quaid-i-Azam University, javidshabbir@gmail.com

Hina Khan

Government College University, hinakhan@gcu.edu.pk

Follow this and additional works at: https://digitalcommons.wayne.edu/jmasm

Part of the Applied Statistics Commons, Social and Behavioral Sciences Commons, and the Statistical Theory Commons

\section{Recommended Citation}

Daraz, Umer; Shabbir, Javid; and Khan, Hina (2018) "Estimation of finite population mean by using minimum and maximum values in stratified random sampling," Journal of Modern Applied Statistical Methods: Vol. 17 : Iss. 1 , Article 20. DOI: $10.22237 /$ jmasm/1532007537

Available at: https://digitalcommons.wayne.edu/jmasm/vol17/iss1/20 


\section{Estimation of finite population mean by using minimum and maximum values in stratified random sampling}

\section{Cover Page Footnote}

Dear Editor, We have revised the manuscript as referees have suggested. 


\section{Estimation of Finite Population Mean by Using Minimum and Maximum Values in Stratified Random Sampling}

\author{
Ummer Draz \\ Quaid-i-Azam University \\ Islamabad, Pakistan
}

\author{
Javid Shabbir \\ Quaid-i-Azam University \\ Islamabad, Pakistan
}

\author{
Hina Khan \\ Government College University \\ Lahore, Pakistan
}

In this paper we have suggested an improved class of ratio type estimators in estimating the finite population mean when information on minimum and maximum values of the auxiliary variable is known. The properties of the suggested class of estimators in terms of bias and mean square error are obtained up to first order of approximation. Two data sets are used for efficiency comparisons.

Keywords: Minimum and maximum values, stratified random sampling, mean squared error, efficiency

\section{Introduction}

In survey sampling, auxiliary information is frequently used in various forms to increase the precision of estimators by taking advantage of the correlation between the study and auxiliary variables. In the proposed class of estimators, the minimum and maximum values of the auxiliary variable are used. Mohanty and Sahoo (1995), Khan and Shabbir (2013), Khan (2015), Walia, Kaur, and Sharma (2015), and Cekim and Cingi (2016) proposed some estimators for the estimation of finite population mean under minimum and maximum values using known information of the auxiliary variable.

Consider a finite population $\mathbf{U}=\left(U_{1}, U_{2}, U_{3}, \ldots, U_{N}\right)$ of size $N$ which is divided into $L$ strata, each of size $N_{\mathrm{h}}(h=1,2, \ldots, L)$, such that $\sum_{h=1}^{L} N_{h}=N$. Let $Y_{h i}$ and $X_{h i}$ be the values of the study variable and the auxiliary variable, respectively, in the $h^{\text {th }}$ stratum of the $i^{\text {th }}$ unit, $i=1,2, \ldots, N_{h}$. We select a sample of size $n_{h}$ from the $h^{\text {th }}$ stratum by using simple random sampling without replacement. Let 


\section{DRAZ ET AL}

$$
\bar{Y}_{h}=\frac{1}{N_{h}} \sum_{i=1}^{N_{h}} Y_{h i} \quad \text { and } \quad \bar{X}_{h}=\frac{1}{N_{h}} \sum_{i=1}^{N_{h}} X_{h i}
$$

be the population means of the study variable $Y$ and the auxiliary variable $X$ in the $h^{\text {th }}$ stratum corresponding to the population means

$$
\bar{Y}=\frac{1}{N_{h}} \sum_{h=1}^{L} W_{h} \bar{Y}_{h} \quad \text { and } \quad \bar{X}=\frac{1}{N_{h}} \sum_{h=1}^{L} W_{h} \bar{X}_{h}
$$

respectively, where $W_{h}=N_{h} / N$ is the known stratum weight. Let

$$
S_{y h}^{2}=\frac{\sum_{i=1}^{N_{h}}\left(Y_{h i}-\bar{Y}_{h}\right)^{2}}{N_{h}-1} \text { and } S_{x h}^{2}=\frac{\sum_{i=1}^{N_{h}}\left(X_{h i}-\bar{X}_{h}\right)^{2}}{N_{h}-1}
$$

be the population variances in the $h^{\text {th }}$ stratum. Let $C_{y h}=S_{y h} / \bar{Y}_{h}$ and $C_{x h}=S_{x h} / \bar{X}_{h}$ be the population coefficients of variations in the $h^{\text {th }}$ stratum. Let $C_{y x h}=\rho_{y x h} C_{y h} C_{x h}$ be the population co-variance between the study variable $(Y)$ and the auxiliary variable $(X)$ in the $h^{\text {th }}$ stratum. Let

$$
\bar{y}_{h}=\frac{1}{n_{h}} \sum_{i=1}^{n_{h}} Y_{h i} \quad \text { and } \quad \bar{x}_{h}=\frac{1}{n_{h}} \sum_{i=1}^{n_{h}} X_{h i}
$$

be the sample means of the study variable $(Y)$ and the auxiliary variable $(X)$ in the $h^{\text {th }}$ stratum. The usual unbiased estimator for the population mean $\bar{Y}$ is given by

$$
\bar{y}_{\mathrm{st}}=\sum_{h=1}^{L} W_{h} \bar{y}_{h}
$$

The variance of $\bar{y}_{\mathrm{st}}$ is given by

$$
\mathrm{V}\left(\bar{y}_{\mathrm{st}}\right)=\sum_{h=1}^{L} W_{h}^{2} \theta_{h} \bar{Y}_{h}^{2} C_{y h}^{2}
$$

where 


\section{ESTIMATION OF FINITE POPULATION MEAN}

$$
\theta_{h}=\left(\frac{1}{n_{h}}-\frac{1}{N_{h}}\right)
$$

The usual ratio estimator for $\bar{Y}$ in stratified random sampling is given by

$$
\bar{y}_{\mathrm{Rs}}=\sum_{h=1}^{L} W_{h} \bar{y}_{h}\left(\frac{\bar{X}_{h}}{\bar{x}_{h}}\right)
$$

The bias and mean square error of $\bar{y}_{\mathrm{Rs}}$ in stratified random sampling are given by

$$
\operatorname{Bias}\left(\bar{y}_{\mathrm{Rs}}\right) \cong \sum_{h=1}^{L} W_{h} \theta_{h} \bar{Y}_{h}\left(C_{x h}^{2}-C_{y s h}\right)
$$

and

$$
\operatorname{MSE}\left(\bar{y}_{\mathrm{Rs}}\right) \cong \sum_{h=1}^{L} W_{h}^{2} \theta_{h} \bar{Y}_{h}^{2}\left(C_{y h}^{2}+C_{x h}^{2}-2 C_{y s h}\right)
$$

The separate linear regression estimator $\bar{y}_{\text {lrs }}$ of the population mean $\bar{Y}$ is given by

$$
\bar{y}_{\mathrm{lrs}}=\sum_{h=1}^{L} W_{h}\left[\bar{y}_{h}+\hat{\beta}_{y x h}\left(\bar{X}_{h}-\bar{x}_{h}\right)\right]
$$

where

$$
\hat{\beta}_{y x h}=\frac{s_{y x h}}{s_{x h}^{2}}
$$

is the sample regression coefficient whose population regression coefficient is $\beta_{y x h}$.

Expressions for bias and mean square error of $\bar{y}_{\text {lrs }}$ are given by

$$
\operatorname{Bias}\left(\bar{y}_{\mathrm{lrs}}\right) \cong \sum_{h=1}^{L} W_{h} \theta_{h} \beta_{y x h} \bar{X}_{h} C_{x h}\left[\lambda_{03 h}-\frac{\lambda_{12 h}}{\rho_{y x h}}\right]
$$

and 


\section{DRAZ ET AL}

$$
\operatorname{MSE}\left(\bar{y}_{\mathrm{lrs}}\right) \cong \sum_{h=1}^{L} W_{h}^{2} \theta_{h} \bar{Y}_{h}^{2} C_{y h}^{2}\left(1-\rho_{y x h}^{2}\right)
$$

where

$$
\lambda_{r s h}=\frac{\mu_{r s h}}{\mu_{02 h}^{r / 2} \mu_{20 h}^{s / 2}}
$$

with

$$
\mu_{r s h}=\frac{\sum_{i=1}^{N_{h}}\left(Y_{h i}-\bar{Y}_{h}\right)^{r}\left(X_{h i}-\bar{X}_{h}\right)^{2}}{N_{h}-1} \text { for } h=1,2,3, \ldots, L
$$

Mohanty and Sahoo (1995) proposed two estimators by using known information of minimum and maximum values of the auxiliary variable. The transformations are given by

$$
v_{h}=\frac{X_{h i}+X_{\mathrm{m} h}}{X_{\mathrm{M} h}+X_{\mathrm{m} h}}
$$

and

$$
z_{h}=\frac{X_{h i}+x_{\mathrm{M} h}}{X_{\mathrm{M} h}+X_{\mathrm{m} h}}
$$

where $X_{\mathrm{m} h}$ and $X_{\mathrm{M} h}$ are the minimum and maximum values of the auxiliary variable in the $h^{\text {th }}$ stratum.

In stratified sampling, the ratio estimators are given by

$$
\bar{y}_{h 1 \mathrm{~s}}=\sum_{h=1}^{L} W_{h} \bar{y}_{h}\left(\frac{\bar{V}_{h}}{\bar{v}_{h}}\right)
$$

and 


\section{ESTIMATION OF FINITE POPULATION MEAN}

$$
\bar{y}_{h 2 \mathrm{~s}}=\sum_{h=1}^{L} W_{h} \bar{y}_{h}\left(\frac{\bar{Z}_{h}}{\bar{z}_{h}}\right)
$$

Expressions for biases and mean squared errors of these estimators are given by

$$
\begin{gathered}
\operatorname{Bias}\left(\bar{y}_{h 1 \mathrm{~s}}\right) \cong \sum_{h=1}^{L} W_{h} \theta_{h} \bar{Y}_{h}\left(t_{1 h}^{2} C_{x h}^{2}-t_{1 h} C_{y x h}\right) \\
\operatorname{Bias}\left(\bar{y}_{h 2 \mathrm{~s}}\right) \cong \sum_{h=1}^{L} W_{h} \theta_{h} \bar{Y}_{h}\left(t_{2 h}^{2} C_{x h}^{2}-t_{2 h} C_{y x h}\right)
\end{gathered}
$$

and

$$
\begin{gathered}
\operatorname{MSE}\left(\bar{y}_{h 1 \mathrm{~s}}\right) \cong \sum_{h=1}^{L} W_{h}^{2} \theta_{h} \bar{Y}_{h}^{2}\left(C_{y h}^{2}-2 t_{1 h} C_{y x h}+t_{1 h}^{2} C_{x h}^{2}\right) \\
\operatorname{MSE}\left(\bar{y}_{h 2 \mathrm{~s}}\right) \cong \sum_{h=1}^{L} W_{h}^{2} \theta_{h} \bar{Y}_{h}^{2}\left(C_{y h}^{2}-2 t_{2 h} C_{y x h}+t_{2 h}^{2} C_{x h}^{2}\right)
\end{gathered}
$$

where

$$
t_{1 h}=\frac{\bar{X}_{h}}{\bar{X}_{h}+X_{\mathrm{m} h}} \quad \text { and } \quad t_{2 h}=\frac{\bar{X}_{h}}{\bar{X}_{h}+X_{\mathrm{M} h}}
$$

Walia et al. (2015) proposed some estimators by using known information on minimum and maximum values of the auxiliary variable. The transformation is given by

$$
z_{1 h}=X_{h i}+\frac{X_{\mathrm{M} h}}{X_{\mathrm{m} h}}
$$

Walia et al. (2015) defined two estimators:

$$
\bar{y}_{\mathrm{m} 1 \mathrm{~s}}=\sum_{h=1}^{L} W_{h} \bar{y}_{h}\left(\frac{\bar{Z}_{1 h}}{\bar{z}_{1 h}}\right)
$$




\section{DRAZ ET AL}

and

$$
\bar{y}_{\mathrm{m} 2 \mathrm{~s}}=\sum_{h=1}^{L} W_{h} \bar{y}_{h}\left(\frac{\bar{Z}_{1 h}+C_{z 1 h}}{\bar{z}_{1 h}+C_{z 1 h}}\right)
$$

where

$$
C_{z 1 h}=\frac{S_{z 1 h}}{\bar{Z}_{1 h}}=\frac{S_{x h}}{\bar{X}_{h}+\frac{X_{\mathrm{M} h}}{X_{\mathrm{m} h}}}
$$

Expressions for biases and mean square errors of these estimators, up to first order approximation, are given by

$$
\begin{aligned}
& \operatorname{Bias}\left(\bar{y}_{\mathrm{m} 1 \mathrm{~s}}\right) \cong \sum_{h=1}^{L} W_{h} \theta_{h} \bar{Y}_{h}\left(C_{1 h}^{2} C_{x h}^{2}-C_{1 h} \rho_{y x h}\right) \\
& \operatorname{Bias}\left(\bar{y}_{\mathrm{m} 2 \mathrm{~s}}\right) \cong \sum_{h=1}^{L} W_{h} \theta_{h} \bar{Y}_{h}\left(C_{2 h}^{2} C_{x h}^{2}-C_{2 h} \rho_{y x h}\right)
\end{aligned}
$$

and

$$
\begin{gathered}
\operatorname{MSE}\left(\bar{y}_{m 1 s}\right) \cong \sum_{h=1}^{L} W_{h}^{2} \theta_{h} \bar{Y}_{h}^{2}\left(C_{y h}^{2}-2 C_{1 h} \rho_{y x h}+C_{1 h}^{2} C_{x h}^{2}\right) \\
\operatorname{MSE}\left(\bar{y}_{m 2 s}\right) \cong \sum_{h=1}^{L} W_{h}^{2} \theta_{h} \bar{Y}_{h}^{2}\left(C_{y h}^{2}-2 C_{2 h} \rho_{y x h}+C_{2 h}^{2} C_{x h}^{2}\right)
\end{gathered}
$$

where

$$
C_{1 h}=\frac{\bar{X}_{h}}{\bar{X}_{h}+\frac{X_{\mathrm{M} h}}{X_{\mathrm{m} h}}} \text { and } C_{2 h}=\frac{\bar{X}_{h}\left(\bar{X}_{h}+\frac{X_{\mathrm{M} h}}{X_{\mathrm{m} h}}\right)}{\left(\bar{X}_{h}+\frac{X_{\mathrm{M} h}}{X_{\mathrm{m} h}}\right)^{2}+S_{x h}}
$$




\section{ESTIMATION OF FINITE POPULATION MEAN}

\section{Proposed Estimator}

Motivated by Koyuncu (2012), an improved class of estimators is proposed for estimating the finite population mean using known information on minimum and maximum values of the auxiliary variable under stratified random sampling. The proposed estimator is given by

$$
\bar{y}_{\mathrm{DS}}=\sum_{h=1}^{L} W_{h}\left[k_{1 h} \bar{y}_{h}\left(\frac{\bar{X}_{h}}{\bar{x}_{h}}\right)^{\alpha_{1}}+k_{2 h}\left(\frac{\bar{X}_{h}}{\bar{x}_{h}}\right)^{\alpha_{2}}\right] \exp \left[\frac{\left(\bar{X}_{h}-\bar{x}_{h}\right)}{\left(\bar{X}_{h}+\bar{x}_{h}\right)+2 j_{h}}\right]
$$

where $k_{i h}, i=1,2$, are unknown constants whose values are to be determined such that the bias and mean square error are minimum, $j_{h}=X_{\mathrm{M} h}-X_{\mathrm{m} h}$, and $\alpha_{1}, \alpha_{2}$ are scalar quantities which contain the values $(0,-1,1)$. From $(24)$, we obtain the different classes of estimators which are given in Table 1.

Table 1. Some classes of estimators in stratified random sampling

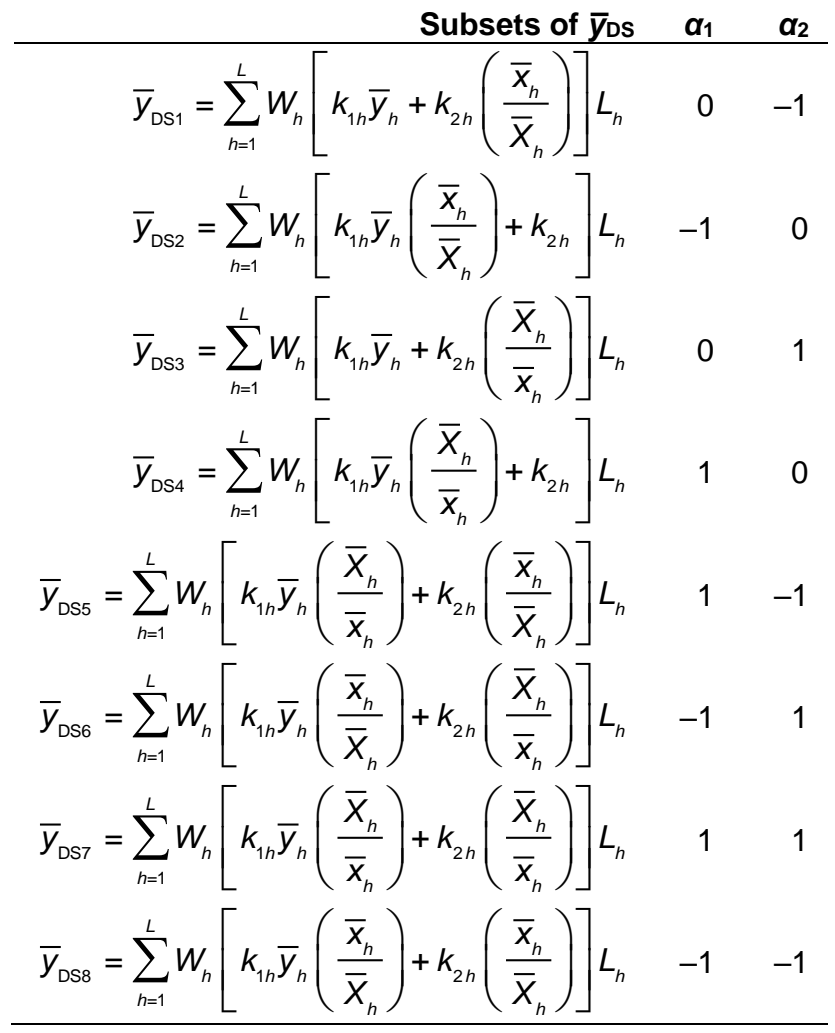




\section{DRAZ ET AL}

where

$$
L_{h}=\exp \left[\frac{\left(\bar{X}_{h}-\bar{x}_{h}\right)}{\left(\bar{X}_{h}+\bar{x}_{h}\right)+2 j_{h}}\right]
$$

To obtain the properties of the proposed estimator, we define the following error terms: Let

$$
e_{0 h}=\left(\frac{\bar{y}_{h}-\bar{Y}_{h}}{\bar{Y}_{h}}\right) \text { and } \quad e_{1 h}=\left(\frac{\bar{x}_{h}-\bar{X}_{h}}{\bar{X}_{h}}\right)
$$

such that $\mathrm{E}\left(e_{i h}\right)=0$ for $i=0,1, \mathrm{E}\left(e_{0 h}^{2}\right)=\theta_{h} C_{y h}^{2}, \quad \mathrm{E}\left(e_{1 h}^{2}\right)=\theta_{h} C_{x h}^{2}$, and $\mathrm{E}\left(e_{0 h} e_{1 h}\right)=\theta_{h} C_{y x h}$.

Rewriting (24) in terms of errors, we have

$$
\bar{y}_{\mathrm{DS}}=\sum_{h=1}^{L} W_{h}\left[k_{1 h} \bar{Y}_{h}\left(1+e_{0 h}\right)\left(1+e_{1 h}\right)^{-\alpha_{1}}+k_{2 h}\left(1+e_{1 h}\right)^{-\alpha_{2}}\right] \exp \left[\frac{-g_{1 h} e_{1 h}}{2}\left(1+\frac{g_{1 h}}{2} e_{1 h}\right)^{-1}\right]
$$

where

$$
g_{1 h}=\frac{\bar{X}_{h}}{\bar{X}_{h}+X_{\mathrm{M} h}-X_{\mathrm{m} h}}
$$

Up to first order of approximation,

$$
\begin{aligned}
\bar{y}_{\mathrm{DS}}-\bar{Y} \cong \sum_{h=1}^{L} W_{h}\left[-\bar{Y}_{h}+k_{1 h} \bar{Y}_{h}\left\{1+e_{0 h}-e_{1 h}\left(\alpha_{1}+\frac{g_{1 h}}{2}\right)\right.\right. \\
\left.+e_{1 h}^{2}\left(\frac{\alpha_{1} g_{1 h}}{2}+\frac{3 g_{1 h}^{2}}{8}+\frac{\alpha_{1}\left(\alpha_{2}+1\right)}{2}\right)-e_{0 h} e_{1 h}\left(\alpha_{1}+\frac{g_{1 h}}{2}\right)\right\} \\
+k_{2 h}\left\{1-e_{1 h}\left(\alpha_{2}+\frac{g_{1 h}}{2}\right)+e_{1 h}^{2}\left(\frac{\alpha_{2} g_{1 h}}{2}+\frac{3 g_{1 h}^{2}}{8}+\frac{\alpha_{2}\left(\alpha_{2}+1\right)}{2}\right)\right\}
\end{aligned}
$$

Using (25), the bias of $\bar{y}_{\mathrm{DS}}$, up to first order of approximation, is given by 


\section{ESTIMATION OF FINITE POPULATION MEAN}

$$
\operatorname{Bias}\left(\bar{y}_{\mathrm{DS}}\right) \cong \sum_{h=1}^{L} W_{h}\left(-\bar{Y}_{h}+k_{1 h} \bar{Y}_{h} D_{h}+k_{2 h} G_{h}\right)
$$

where

$$
D_{h}=\left[1+\theta_{h} C_{x h}^{2}\left(\frac{\alpha_{1} g_{1 h}}{2}+\frac{3 g_{1 h}^{2}}{8}+\frac{\alpha_{1}\left(\alpha_{1}+1\right)}{2}\right)-\theta_{h} C_{y x h}\left(\alpha_{1}+\frac{g_{1 h}}{2}\right)\right]
$$

and

$$
G_{h}=\left[1+\theta_{h} C_{x h}^{2}\left(\frac{\alpha_{2} g_{1 h}}{2}+\frac{3 g_{1 h}^{2}}{8}+\frac{\alpha_{2}\left(\alpha_{2}+1\right)}{2}\right)\right]
$$

By squaring and taking expectations on both sides of (25), obtain the mean square error up to first order of approximation, which is given by

$$
\begin{aligned}
\operatorname{MSE}\left(\bar{y}_{\mathrm{DS}}\right) \cong \sum_{h=1}^{L} W_{h}^{2}\left(\bar{Y}_{h}^{2}+\bar{Y}_{h}^{2} k_{1 h}^{2} A_{h}+k_{2 h}^{2} B_{h}-2 \bar{Y}_{h}^{2} k_{1 h} D_{h}\right. \\
\left.-2 \bar{Y}_{h} k_{2 h} G_{h}+2 \bar{Y}_{h} k_{1 h} k_{2 h} F_{h}\right)
\end{aligned}
$$

where

$$
\begin{gathered}
A_{h}=\left[1+\theta_{h}\left\{C_{y h}^{2}+C_{x h}^{2}\left\{\left(\alpha_{1}+\frac{g_{1 h}}{2}\right)^{2}+\left(\alpha_{1} g_{1 h}+\frac{3 g_{1 h}^{2}}{4}+\alpha_{1}\left(\alpha_{1}+1\right)\right)\right\}\right.\right. \\
\left.\left.-4 C_{y x h}\left(a_{1}+\frac{g_{1 h}}{2}\right)\right\}\right] \\
B_{h}=\left[1+\theta_{h} C_{x h}^{2}\left\{\left(\alpha_{2}+\frac{g_{1 h}}{2}\right)^{2}+\left(\alpha_{2} g_{1 h}+\frac{3 g_{1 h}^{2}}{4}+\alpha_{2}\left(\alpha_{2}+1\right)\right)\right\}\right]
\end{gathered}
$$

and 


$$
\begin{aligned}
F_{h}=\left[1+\theta_{h}\left\{C_{x h}^{2}\right.\right. & \left(\frac{\alpha_{1} g_{1 h}}{2}+\frac{3 g_{1 h}^{2}}{8}+\frac{\alpha_{1}\left(\alpha_{1}+1\right)}{2}\right)+\left(\alpha_{1}+\frac{g_{1 h}}{2}\right)\left(\alpha_{2}+\frac{g_{1 h}}{2}\right) \\
& \left.\left.\left.+\left(\frac{\alpha_{2} g_{1 h}}{2}+\frac{3 g_{1 h}^{2}}{8}+\frac{\alpha_{2}\left(\alpha_{2}+1\right)}{2}\right)\right\}-C_{y x h}\left(\alpha_{1}+\alpha_{2}+g_{1 h}\right)\right\}\right]
\end{aligned}
$$

The optimum values of $k_{1 h}$ and $k_{2 h}$ obtained by minimizing (27) are, respectively, given by

$$
k_{1 h(\mathrm{opt})}=\frac{B_{h} D_{h}-F_{h} G_{h}}{A_{h} B_{h}-F_{h}^{2}} \quad \text { and } \quad k_{2 h(\mathrm{opt})}=\frac{\bar{Y}_{h}\left(A_{h} G_{h}-D_{h} F_{h}\right)}{A_{h} B_{h}-F_{h}^{2}}
$$

Substituting the optimum values of $k_{1 h}$ and $k_{2 h}$ into (26) and (27) yields the minimum bias and MSE of $\bar{y}_{\mathrm{DS}}$, given by

$$
\operatorname{Bias}\left(\bar{y}_{\mathrm{DS}}\right)_{\min } \cong-\sum_{h=1}^{L} W_{h} \bar{Y}_{h}\left[1-\frac{\left(A_{h} G_{h}^{2}+B_{h} D_{h}^{2}-2 D_{h} F_{h} G_{h}\right)}{A_{h} B_{h}-F_{h}^{2}}\right]
$$

and

$$
\operatorname{MSE}\left(\bar{y}_{\mathrm{DS}}\right)_{\min } \cong \sum_{h=1}^{L} W_{h}^{2} \bar{Y}_{h}^{2}\left[1-\frac{\left(A_{h} G_{h}^{2}+B_{h} D_{h}^{2}-2 D_{h} F_{h} G_{h}\right)}{A_{h} B_{h}-F_{h}^{2}}\right]
$$

\section{Comparison of Estimators}

Now, compare the proposed class of estimators $\bar{y}_{\mathrm{DS}}$ with existing estimators $\bar{y}_{\mathrm{st}}, \bar{y}_{\mathrm{Rs}}$, $\bar{y}_{\mathrm{rrs}}, \bar{y}_{h 1 \mathrm{~s}}, \bar{y}_{h 2 \mathrm{~s}}, \bar{y}_{\mathrm{m} 1 \mathrm{~s}}$, and $\bar{y}_{\mathrm{m} 2 \mathrm{~s}}$.

Condition (i) By (2) and (29), $\mathrm{V}\left(\overline{\bar{y}}_{\mathrm{st}}\right)>\operatorname{MSE}\left(\bar{y}_{\mathrm{DS}}\right)_{\min }$ if

$$
\sum_{h=1}^{L} W_{h}^{2} \bar{Y}_{h}^{2}\left[\theta_{h} C_{y h}^{2}-1+\frac{\left(B_{h} D_{h}^{2}+A_{h} G_{h}^{2}-2 D_{h} F_{h} G_{h}\right)}{A_{h} B_{h}-F_{h}^{2}}\right]>0
$$




\section{ESTIMATION OF FINITE POPULATION MEAN}

Condition (ii) $\quad$ By (5) and (29), $\operatorname{MSE}\left(\bar{y}_{\mathrm{Rs}}\right)>\operatorname{MSE}\left(\bar{y}_{\mathrm{DS}}\right)_{\min }$ if

$$
\sum_{h=1}^{L} W_{h}^{2} \bar{Y}_{h}^{2}\left[\theta_{h}\left(C_{y h}^{2}+C_{x h}^{2}-2 C_{y x h}\right)-1+\frac{\left(B_{h} D_{h}^{2}+A_{h} G_{h}^{2}-2 D_{h} F_{h} G_{h}\right)}{A_{h} B_{h}-F_{h}^{2}}\right]>0
$$

Condition (iii) $\quad$ By (8) and (29), $\operatorname{MSE}\left(\bar{y}_{\mathrm{lrs}}\right)_{\min }>\operatorname{MSE}\left(\bar{y}_{\mathrm{DS}}\right)_{\min }$ if

$$
\sum_{h=1}^{L} W_{h}^{2} \bar{Y}_{h}^{2}\left[\theta_{h} C_{y h}^{2}\left(1-\rho_{y x h}^{2}\right)-1+\frac{\left(B_{h} D_{h}^{2}+A_{h} G_{h}^{2}-2 D_{h} F_{h} G_{h}\right)}{A_{h} B_{h}-F_{h}^{2}}\right]>0
$$

Condition (iv) $\quad \mathrm{By}(15)$ and (29), $\operatorname{MSE}\left(\bar{y}_{h 1 \mathrm{~s}}\right)>\operatorname{MSE}\left(\overline{\bar{y}}_{\mathrm{DS}}\right)_{\min }$ if

$$
\sum_{h=1}^{L} W_{h}^{2} \bar{Y}_{h}^{2}\left[\theta_{h}\left(C_{y h}^{2}+t_{1 h}^{2} C_{x h}^{2}-2 t_{1 h} C_{y x h}\right)-1+\frac{\left(B_{h} D_{h}^{2}+A_{h} G_{h}^{2}-2 D_{h} F_{h} G_{h}\right)}{A_{h} B_{h}-F_{h}^{2}}\right]>0
$$

Condition (v) By (16) and (29), $\operatorname{MSE}\left(\bar{y}_{h 2 \mathrm{~s}}\right)>\operatorname{MSE}\left(\bar{y}_{\mathrm{DS}}\right)_{\min }$ if

$$
\sum_{h=1}^{L} W_{h}^{2} \bar{Y}_{h}^{2}\left[\theta_{h}\left(C_{y h}^{2}+t_{2 h}^{2} C_{x h}^{2}-2 t_{2 h} C_{y x h}\right)-1+\frac{\left(B_{h} D_{h}^{2}+A_{h} G_{h}^{2}-2 D_{h} F_{h} G_{h}\right)}{A_{h} B_{h}-F_{h}^{2}}\right]>0
$$

Condition (vi) By (22) and (29), $\operatorname{MSE}\left(\bar{y}_{\mathrm{m} 1 \mathrm{~s}}\right)>\operatorname{MSE}\left(\bar{y}_{\mathrm{DS}}\right)_{\min }$ if

$$
\sum_{h=1}^{L} W_{h}^{2} \bar{Y}_{h}^{2}\left[\theta_{h}\left(C_{y h}^{2}+C_{1 h}^{2} C_{x h}^{2}-2 C_{1 h} C_{y x h}\right)-1+\frac{\left(B_{h} D_{h}^{2}+A_{h} G_{h}^{2}-2 D_{h} F_{h} G_{h}\right)}{A_{h} B_{h}-F_{h}^{2}}\right]>0
$$

Condition (vii) By (23) and (29), $\operatorname{MSE}\left(\bar{y}_{\mathrm{m} 2 \mathrm{~s}}\right)>\operatorname{MSE}\left(\bar{y}_{\mathrm{DS}}\right)_{\min }$ if

$$
\sum_{h=1}^{L} W_{h}^{2} \bar{Y}_{h}^{2}\left[\theta_{h}\left(C_{y h}^{2}+C_{2 h}^{2} C_{x h}^{2}-2 C_{2 h} C_{y x h}\right)-1+\frac{\left(B_{h} D_{h}^{2}+A_{h} G_{h}^{2}-2 D_{h} F_{h} G_{h}\right)}{A_{h} B_{h}-F_{h}^{2}}\right]>0
$$




\section{Numerical Examples}

Two data sets are used to obtain the percent relative efficiency (PRE) of the different estimators:

Data Set 1. (Bureau of Statistics, 2013, p. 226)

$Y$ : Employment level in 2012 by divisions.

$X$ : Number of registered factories in 2012 by divisions.

The data are divided into two groups:

Group 1: Gujranwala, Lahore, Rawalpindi, and Sargodha divisions.

Group 2: Bahawalpur, D.G Khan, Faisalabad, Multan, and Sahiwal divisions.

The summary statistics are given below:

$$
\begin{aligned}
& N_{1}=18, N_{2}=18, n_{1}=8, n_{2}=8, \bar{Y}_{1}=85572.11, \overline{Y_{2}}=19293.61, \bar{X}_{1}=414.5556, \\
& \bar{X}_{2}=257, \quad X_{\mathrm{M} 1}=2055, \quad X_{\mathrm{M} 2}=1674, \quad X_{\mathrm{m} 1}=24, \quad X_{\mathrm{m} 2}=52, \quad S_{y 1}=248216, \\
& S_{y 2}=37979.33, S_{x 1}=521.6751, S_{x 2}=365.6955, \rho_{y x 1}=0.3473, \rho_{y x 2}=0.9796, \\
& C_{x 1}=1.2584, C_{x 2}=1.423, C_{y 1}=2.9007, C_{y 2}=1.9685
\end{aligned}
$$

Data Set 2. (Bureau of Statistics, 2013, p. 135)

$Y$ : Total number of students enrolled in 2012 by divisions.

$X$ : Total number of government primary and secondary schools for boys and girls in 2012 by divisions.

The data are divided into two groups:

Group 1: Gujranwala, Lahore, Rawalpindi, and Sargodha divisions.

Group 2: Bahawalpur, D.G Khan, Faisalabad, Multan, and Sahiwal divisions.

The summary statistics are given below:

$$
\begin{aligned}
& N_{1}=18, N_{2}=18, n_{1}=8, n_{2}=8, \bar{Y}_{1}=162979.3, \bar{Y}_{2}=134458, \bar{X}_{1}=962.0556, \\
& \bar{X}_{2}=1146.722, \quad S_{y 1}=255887.7, \quad S_{y 2}=50235.82, \quad S_{x 1}=307.9531, \\
& S_{x 2}=469.9311, X_{\mathrm{M} 1}=1530, X_{\mathrm{M} 2}=2370, X_{\mathrm{m} 1}=388, X_{\mathrm{m} 2}=582, \rho_{y x 1}=0.1447, \\
& \rho_{y x 2}=0.787, C_{x 1}=0.3202, C_{x 2}=0.4098, C_{y 1}=1.5701, C_{y 2}=0.3736
\end{aligned}
$$




\section{ESTIMATION OF FINITE POPULATION MEAN}

Table 2. Percent relative efficiency of different estimators with respect to $\bar{y}_{s t}$

\begin{tabular}{rrr} 
Estimator & Data set 1 & Data set 2 \\
\hline $\bar{y}_{\text {st }}$ & 100.000 & 100.000 \\
$\bar{y}_{\mathrm{Rs}}$ & 115.073 & 103.756 \\
$\bar{y}_{\mathrm{rrs}}$ & 116.252 & 104.509 \\
$\bar{y}_{h 1 \mathrm{~s}}$ & 115.298 & 104.495 \\
$\bar{y}_{h 2 \mathrm{~s}}$ & 105.080 & 103.318 \\
$\bar{y}_{\mathrm{m} 1 \mathrm{~s}}$ & 115.883 & 103.776 \\
$\bar{y}_{\mathrm{m} 2 \mathrm{~s}}$ & 115.880 & 103.778 \\
$\bar{y}_{\mathrm{DS} 1}$ & 686.109 & 2142.170 \\
$\bar{y}_{\mathrm{DS} 2}$ & 131154.840 & 49578.150 \\
$\bar{y}_{\mathrm{DS} 3}$ & 1106.640 & 1724.010 \\
$\bar{y}_{\mathrm{DS} 4}$ & 75600.490 & 29063.170 \\
$\bar{y}_{\mathrm{DS} 5}$ & 893.565 & 3726.730 \\
$\bar{y}_{\mathrm{DS} 6}$ & 2145.380 & 2142.520 \\
$\bar{y}_{\mathrm{DS} 7}$ & 770.943 & 1235.560 \\
$\bar{y}_{\mathrm{DS} 8}$ & 782.772 & 2920.860 \\
\hline
\end{tabular}

We use the following expression for efficiency comparisons:

$$
P R E=\frac{\mathrm{V}\left(\bar{y}_{\mathrm{st}}\right)}{\operatorname{MSE}\left(\bar{y}_{k}\right)} \times 100
$$

where $k$ is one of Rs, lrs, $h 1 \mathrm{~s}, h 2 \mathrm{~s}, \mathrm{~m} 1 \mathrm{~s}, \mathrm{~m} 2 \mathrm{~s}$, or DSi $(i=1,2, \ldots, 8)$. The percent relative efficiencies are summarized for two data sets in Table 2.

\section{Conclusion}

An improved class of estimators was proposed in estimating the finite population mean using known minimum and maximum values of the auxiliary variable. Shown in Table 2 are the percent relative efficiencies of all estimators over the usual mean per unit estimator $\bar{y}_{\mathrm{st}}$ in stratified random sampling. It was observed that the performance of the suggested class of estimators $\overline{y_{\mathrm{DS}} i}(i=1,2, \ldots, 8)$ is better as compared to all other considered estimators. Among the suggested class of estimators, $\bar{y}_{\mathrm{DS} 2}$ is preferable because of least MSE.

\section{References}

Bureau of Statistics. (2013). Punjab development statistics. Lahore, Pakistan: Bureau of Statistics, Government of the Punjab. Retrieved from http://www.bos.gop.pk/system/files/Dev-2013.pdf 


\section{DRAZ ET AL}

Cekim, H. O., \& Cingi, H. (2016). Some estimator types for population mean using linear transformation with the help of the minimum and maximum values of the auxiliary variable. Hacettepe Journal of Mathematics and Statistics, 46(4). doi: 10.15672/hjms.201510114186

Khan, M. (2015). Improvement in estimating the finite population mean under maximum and minimum values in double sampling scheme. Journal of Statistics Applications \& Probability Letters, 2(2), 115-121. Retrieved from http://www.naturalspublishing.com/ContIss.asp?IssID=262

Khan, M., \& Shabbir, J. (2013). Some improved ratio, product, and regression estimators of finite population mean when using minimum and maximum values. The Scientific World Journal, 2013, 431868. doi:

$10.1155 / 2013 / 431868$

Koyuncu, N. (2012). Efficient estimators of population mean using auxiliary attributes. Applied Mathematics and Computation, 218(22), 10900-10905. doi: 10.1016/j.amc.2012.04.050

Mohanty, S., \& Sahoo, J. (1995). A note on improving the ratio method of estimation through linear transformation using certain known population parameters. Sankhyā: The Indian Journal of Statistics, Series B, 57(1), 93-102. Available from https://www.jstor.org/stable/25052879

Walia, G. S., Kaur, H., \& Sharma, M. (2015). Ratio type estimator of population mean through efficient linear transformation. American Journal of Mathematics and Statistics, 5(3), 144-149. Retrieved from http://article.sapub.org/10.5923.j.ajms.20150503.06.html 